\title{
Integer Tree-Based Search and Mixed-Integer Optimal Control of Distribution Chains
}

\author{
A. Alessandri, M. Gaggero, and F. Tonelli
}

\begin{abstract}
The use of integer tree-based search and mixedinteger programming is investigated for the purpose of control of multi-item multi-echelon distribution chains. A discrete-time model is considered to describe the dynamics of a generic distribution chain. The decisions on the amounts of goods to transfer are made by referring to a performance index that accounts for transportation, storage, and backlog costs at two levels, i.e., strategic and tactical. As to the strategic level, a worst-case stock replenishment policy is adopted to exploit the uncertain information available on long-term predictions of the customers' demand. The solution of such a problem is obtained by using a top-down tree-based algorithm to select policy parameters such as the delivery cycle times of goods. At the tactical level, the on-line decisions on the transportation of goods are taken basing on model predictive control, which allows one to take into account recent, reliable, short-term predictions of the demand. The tactical optimal decisions are obtained by solving mixed-integer programming problems with fewer variables as compared with the strategic setting. Simulation results are presented to show the effectiveness of the proposed approach.
\end{abstract}

\section{INTRODUCTION}

In this paper, we focus on integer programming problems that arise when dealing with the management of distribution chains (DCs), where the uncertainty on the customers' demand varies over time, i.e., it is large at first and much lower during on-line operations, when some (but not all) decisions can be somehow refined to improve the performances.

A DC is a complex system that is driven by the external demand and depends on a large number of decision variables. Such decisions concern the delivery of goods up to destination throughout the network and are made on the basis of the information available on the customers' requests. The management of a DC consists in planning the flows of goods while trading between transportation/inventory costs and satisfying the customers' demand. As the degree of uncertainty on the demand predictions may be large at first but becomes lower when the decisions are taken during operations, a twolevel hierarchical approach is proposed in [1]. In the first level, off-line strategic decisions are taken basing on the uncertainty on the available long-term information. In the second level, tactical decisions are determined on line during operations at each time instant when more precise, shortterm predictions of the customers' demand become available. Basing on the findings of [1], in this paper we focus on the use of integer tree-based and mixed-integer optimal control

A. Alessandri and F. Tonelli are with the Department of Production Engineering, Thermoenergetics, and Mathematical Models, University of Genoa, Italy (e-mails: \{alessandri, flavio.tonelli\}@diptem.unige.it). M. Gaggero is with the Institute of Intelligent Systems for Automation, National Research Council of Italy, Italy (e-mail: mauro.gaggero@ge.issia.cnr.it). that are conveniently combined to solve the strategic and tactical problems.

At the strategic level, the information on the customers' demand is of long-term type and so quite poor, thus entailing a conservative design, for which a min-max approach is proposed. At the tactical level, the decisions that can be changed during operations are tuned by using the new, shortterm, and more precise predictions of the demands that become available. This task is accomplished by using model predictive control (MPC), which allows one to take advantage of the information available on the future behavior of the system [2]-[6]. In our context, as we shall see, there exists the need of dealing with both real and integer variables and hence of addressing mixed-integer optimal control problems. Clearly, the main difficulty to deal with is the computational complexity, as such problems are in general NP-hard [7]. Efficient solution methods based on continuous relaxation are available for quadratic programming (see, e.g., [8], [9] and the references therein). Unfortunately, such approaches are not applicable here since we need to account for nonquadratic cost functions. However, heuristic relaxation techniques were successfully proposed in [1].

In this paper, novel contributions in terms of algorithmic improvements are proposed as compared with [1]. Both in the strategic and tactical settings, the discontinuous, stepincreasing nature of the cost function to be optimized is taken into account via binary variables corresponding to the various steps. Thus, mixed-integer programming techniques have to be used to compute the optimal policies. In the strategic setting, besides such binary variables, additional integer variables are introduced that correspond to the delivery cycle times of goods. In order to deal with such variables, here a new tree-based approach is presented to solve the strategic control problem that allows one to determine the optimal values of safety stocks, delivery cycle times, and forecast flows of goods to transfer in such a way to minimize a given performance index and satisfy exactly the external demand. More specifically, a top-down tree-based algorithm is adopted to deal with the selection of the integer variables that represent the delivery cycle times. The tree is constructed by means of a branching on the choice of such variables. The nodes are associated with potential instances of subproblems that are solved only at the leaves, where the solutions of min-max mixed-integer optimization problems are explicitly computed. Likewise in a branch-and-bound setting, the current best result obtained in solving such problems is used to discriminate among the new solutions that are generated step by step in the construction of the tree. Moreover, one 
can prune the nodes that correspond to problems that are evaluated as infeasible on the basis of previous infeasibility results, thus reducing the computational burden.

The rest of the paper is organized as follows. Section II provides a brief account of the DC model presented in [1]. The novel contributions are reported in Sections III and IV for the strategic and tactical control methodologies, respectively. Simulation results are shown in Section V.

Throughout the paper, we shall denote the real numbers equal to or greater than zero by $\mathbb{R}_{+}$and the positive integer numbers equal to or greater than one by $\mathbb{N}$. Given the real vectors $\underline{e}_{i}, \underline{e}_{i+1}, \ldots, \underline{e}_{i+j}$, we shall adopt the notation $\underline{e}_{i}^{i+j}$ to denote an ordered collection of such vectors given by $\operatorname{col}\left(\underline{e}_{i}, \underline{e}_{i+1}, \ldots \underline{e}_{i+j}\right), i \in \mathbb{N} \cup\{0\}, j \in \mathbb{N}$.

\section{Dynamic Model of Distribution Chains}

Let us focus on a state-space dynamic model of the various flows of goods inside a given DC. In line with [1], we refer to a generic DC composed of production units (PUs), distribution units (DUs), and sales units (SUs). Each unit is represented by a node of a directed graph. A node is equipped with a set of buffers that are filled up with quantities of goods of various types among $M$ possible ones. Given a graph with $N-1$ nodes, we denote the set of the indexes of the types of goods available at node $i$ by $K(i), i=1,2, \ldots, N-1$, and by $I(i)$ and $O(i)$ the sets of the incoming and outgoing neighbors of node $i$, respectively. Moreover, $\tau_{i j k} \in \mathbb{N}$ is the delay required to transfer goods of type $k$ from node $i$ to node $j ; I_{k}(i) \triangleq\{j \in I(i): k \in K(i) \cap K(j)\}$ is the set of the indexes of the types of goods that can be transferred to node $i ; O_{k}(i) \triangleq\{j \in O(i): k \in-K(i) \cap K(j)\}$ is the set of the indexes of the types of goods that can be transferred from node $i$. For the sake of brevity, let $\mathcal{P}, \mathcal{D}$, and $\mathcal{S}$ be the sets of the indexes of the nodes corresponding to PUs, DUs, and SUs, respectively. We denote the initial source at the PUs and final sink for the SUs by the fictitious nodes 0 and $N$, respectively.

According to [1], a DC state-space model can be derived by balancing the stock levels in all the buffers and backlogs at the SU nodes, all regarded as continuous state variables of the following discrete-time dynamic equations:

$$
\begin{aligned}
z_{i k}(t+1) & =z_{i k}(t)+u_{0 i k}\left(t-\tau_{0 i k}\right)-\sum_{j \in O_{k}(i)} u_{i j k}(t) \\
i & \in \mathcal{P}, \quad k \in K(i) \\
z_{i k}(t+1) & =z_{i k}(t)+\sum_{j \in I_{k}(i)} u_{j i k}\left(t-\tau_{j i k}\right)-\sum_{j \in O_{k}(i)} u_{i j k}(t), \\
i & \in \mathcal{D}, \quad k \in K(i) \\
z_{i k}(t+1) & =z_{i k}(t)+\sum_{j \in I_{k}(i)} u_{j i k}\left(t-\tau_{j i k}\right)-u_{i N k}(t) \\
i & \in \mathcal{S}, \quad k \in K(i) \\
b_{i k}(t+1) & =b_{i k}(t)-u_{i N k}(t)+d_{i k}(t) \\
i & \in \mathcal{S}, \quad k \in K(i)
\end{aligned}
$$

where $t=0,1, \ldots ; z_{i k}(t) \in \mathbb{R}$ is the stock level of the buffer that contains quantities of goods of type $k$ at node $i$, $i=1,2, \ldots, N-1, k \in K(i)$ (such variables will be called z-variables); $u_{i j k}(t) \in \mathbb{R}$ is the amount of goods of type $k$ transferred from node $i$ to node $j, i \in I(j), j=1,2, \ldots, N$, $k \in K(i) \cap K(j) ; b_{i k}(t) \in \mathbb{R}$ is the backlog level of the buffer that contains quantities of goods of type $k$ at node $i$, $i \in \mathcal{S}, k \in K(i)$ (such variables will be called b-variables); $d_{i k}(t) \in \mathbb{R}$ is the external demand of goods of type $k$ at node $i, i \in \mathcal{S}, k \in K(i)$.

For the sake of brevity, let us define the state, control, and demand vectors of the overall DC as follows:

$$
\begin{gathered}
\underline{x}_{t} \triangleq \operatorname{col}\left[z_{i k}(t), u_{j i l}(t-1), \ldots, u_{j i l}\left(t-\tau_{j i l}\right),\right. \\
b_{r k}(t), i=1,2, \ldots, N-1, j \in I(i), \\
k \in K(i), l \in K(i) \cap K(j), r \in \mathcal{S}] \in \mathbb{R}^{n} \\
\underline{u}_{t} \triangleq \operatorname{col}\left[u_{i j k}(t), i \in I(j), j=1,2, \ldots, N,\right. \\
k \in K(i) \cap K(j)] \in \mathbb{R}^{m} \\
\underline{d}_{t} \triangleq \operatorname{col}\left[d_{i k}(t), i \in \mathcal{S}, k \in K(i)\right] \in \mathbb{R}^{p} .
\end{gathered}
$$

In order to complete such a model, we need to introduce various types of constraints that stem from both boundedness of the state variables and other limitations concerning the control inputs.

More specifically, first let us consider the state variables. We constrain the stock levels as follows:

$$
0 \leq z_{i k}(t) \leq z_{i k}^{\max }, \quad i=1,2, \ldots, N-1, \quad k \in K(i)
$$

where $t=0,1, \ldots$ and $z_{i k}^{\max }$ is a positive constant representing the maximum quantity of goods of type $k$ that can be stored at node $i, i=1,2, \ldots, N-1, k \in K(i)$. As regards the backlogs, we impose the following constraints:

$$
b_{i k}(t) \geq 0, \quad i \in \mathcal{S}, \quad k \in K(i)
$$

where $t=0,1, \ldots$ Let $\underline{b}_{t} \triangleq \operatorname{col}\left[b_{i k}(t), i \in \mathcal{S}, k \in K(i)\right] \in$ $\mathbb{R}^{p}$. In order to prevent from the occurrence of stockouts, a safety stock may be kept to satisfy unexpected requests. Thus, let $s_{i k} \geq 0$ be the safety stock level at node $i$ for goods of type $k$. From a strategic point of view, we constrain each stock level to be higher than the corresponding safety stock:

$$
z_{i k}(t) \geq s_{i k}, \quad i=1,2, \ldots, N-1, \quad k \in K(i)
$$

where $t=0,1, \ldots$. As we shall see in the following, during on-line operations the constraints (4) can be relaxed, as such stocks are employed on demand to avoid stockouts. Let $\underline{s} \triangleq$ $\operatorname{col}\left[s_{i k}, i=1,2, \ldots, N-1, k \in K(i)\right] \in \mathbb{R}^{q}$.

The control inputs are constrained to satisfy limitations that vary over time. More specifically, since the transportation of goods of type $k$ from node $i$ to node $j$ can be made only with a delivery cycle time denoted by $\Omega_{i j k} \in \mathbb{N}$, the following constraints hold:

$$
\begin{aligned}
& u_{i j k}^{\min }(t) \leq u_{i j k}(t) \leq u_{i j k}^{\max }(t), \\
& i \in I(j), \quad j=1,2, \ldots, N-1, \quad k \in K(i) \cap K(j)
\end{aligned}
$$

where $t=l \Omega_{i j k}, l \in \mathbb{N} ; u_{i j k}^{\min }(t)$ and $u_{i j k}^{\max }(t)$ are positive numbers representing the minimum and maximum amounts of goods of type $k$ that can be transferred at time $t$ from node $i$ to node $j$, respectively. Note that we do not explicitly 
constrain the controls $u_{i N k}(t), i \in \mathcal{S}, k \in K(i), t=0,1, \ldots$, as they model the quantities of goods that leave the SU nodes upon the demands $d_{i k}(t)$. Let $\underline{u}_{\min , t} \triangleq \operatorname{col}\left[u_{i j k}^{\min }(t), i \in\right.$ $I(j), j=1,2, \ldots, N-1, k \in K(i) \cap K(j)] \in \mathbb{R}^{m^{\prime}}$ and $\underline{u}_{\max , t} \triangleq \operatorname{col}\left[u_{i j k}^{\max }(t), i \in I(j), j=1,2, \ldots, N-1\right.$, $k \in K(i) \cap K(j)] \in \mathbb{R}^{m^{\prime}}$.

In order to model the impossibility of transferring goods of type $k$ from node $i$ to node $j$ in time instants that are not multiples of the delivery cycle time $\Omega_{i j k}$, we impose

$$
\begin{aligned}
& u_{i j k}(t)=0, \\
& i \in I(j), \quad j=1,2, \ldots, N-1, \quad k \in K(i) \cap K(j)
\end{aligned}
$$

where $t \neq l \Omega_{i j k}, l \in \mathbb{N}$. In other words, if $\bar{t}=l \Omega_{i j k}, l \in \mathbb{N}$, we constrain $u_{i j k}(t)=0$ for all $t=\bar{t}+1, \bar{t}+2, \ldots, \bar{t}+\Omega_{i j k}-1$. Let $\underline{\Omega} \triangleq$ $\operatorname{col}\left[\Omega_{i j k}, i \in I(j), j=1,2, \ldots, N-1, k \in K(i) \cap K(j)\right] \in$ $\mathbb{N}^{m^{\prime}}$ be the vector of all the delivery cycle times.

\section{Strategic Control of DCs via Tree-BASED Min-MAX Mixed-INTEger Programming}

In this section, we address the problem that consists in choosing the values of safety stocks, delivery cycle times, and forecast flows of goods to transfer according to a worstcase criterion and satisfying exactly the external demands. We shall consider a long time horizon $T$ and a suitable cost function that measures the performance of the DC over such a period of time.

If the initial state $\underline{x}_{0}$ is assigned, the future behavior of the DC up to the time $T$ is determined via the state equation (1), demands $\underline{d}_{0}^{T-1}$, control inputs $\underline{u}_{0}^{T-1}$, safety stocks $\underline{s}$, and delivery cycle times $\underline{\Omega}$. Since satisfying exactly the external demands is equivalent to keep the backlogs at the zero level, we impose the following constraint:

$$
\underline{b}_{t}=\underline{0}, \quad t=1,2, \ldots, T \text {. }
$$

We assume to know a prediction $\underline{\hat{d}}_{t} \in \mathbb{R}^{p}$ of $\underline{d}_{t}$, i.e., $\underline{d}_{t}=\underline{\hat{d}}_{t}+\underline{\tilde{d}}_{t}, t=0,1, \ldots, T-1$, where $\underline{\tilde{d}}_{t} \in \mathbb{R}^{p}$ is the vector of prediction errors: it represents the amount of uncertainty in the prediction of $\underline{d}_{t}$. We suppose to be able to predict the behavior of the demand over time within a given uncertainty band described by known lower and upper bounds, i.e., $\underline{\tilde{d}}_{\min , t} \in \mathbb{R}^{p}$ and $\underline{\tilde{d}}_{\max , t} \in \mathbb{R}^{p}$ such that $\underline{\tilde{d}}_{\min , t} \leq \underline{\tilde{d}}_{t} \leq \underline{\tilde{d}}_{\max , t}, t=0,1, \ldots, T-1$.

Moreover, one may constrain the stock levels at the final time instant to belong to an assigned subset of values:

$$
z_{i k}^{\text {down }} \leq z_{i k}(T) \leq z_{i k}^{\text {up }}, i=1,2, \ldots, N-1, k \in K(i)
$$

where $z_{i k}^{\text {down }}$ and $z_{i k}^{\text {up }}$ are the lower and upper bounds of the corresponding z-variable at time $T$, respectively.

Generally speaking, the goal of the strategic control is to minimize the inventory and transportation costs and satisfy the customers' demands as well. As to the inventory, the higher the amounts of goods stocked at the various nodes, the higher the storage costs. As to the transportation, once fixed the types of carriers, the transportation costs grow if the quantities of transferred goods increase. In [1], the costs related to the transportation of goods from node to node were modeled by a step-increasing function $g_{i j k}: \mathbb{R} \rightarrow \mathbb{R}$, $i \in I(j), j=1,2, \ldots, N-1, k \in K(i) \cap K(j)$, as follows:

$$
\begin{aligned}
& g_{i j k}\left[u_{i j k}(t)\right]= \begin{cases}w_{i j k}^{0} \triangleq 0 & \text { if } u_{i j k}(t)=u_{i j k}^{0} \triangleq 0 \\
w_{i j k}^{1} & \text { if } u_{i j k}^{0}<u_{i j k}(t) \leq u_{i j k}^{1} \\
& \vdots \\
w_{i j k}^{r} & \text { if } u_{i j k}(t)>u_{i j k}^{r-1}\end{cases} \\
& i \in I(j), \quad j=1,2, \ldots, N-1, \quad k \in K(i) \cap K(j)
\end{aligned}
$$

where $w_{i j k}^{0} \leq w_{i j k}^{1} \leq w_{i j k}^{2} \leq \cdots \leq w_{i j k}^{r}, u_{i j k}^{0} \leq u_{i j k}^{1} \leq$ $u_{i j k}^{2} \leq \cdots \leq u_{i j k}^{r-1}$ (see Fig. 1), and $t=0,1, \ldots, T-1$. Such a function is motivated by the fact that, in general, there exists no perfect proportionality between costs and transferred quantities, as the transportation is usually accomplished by lot-sized carriers.

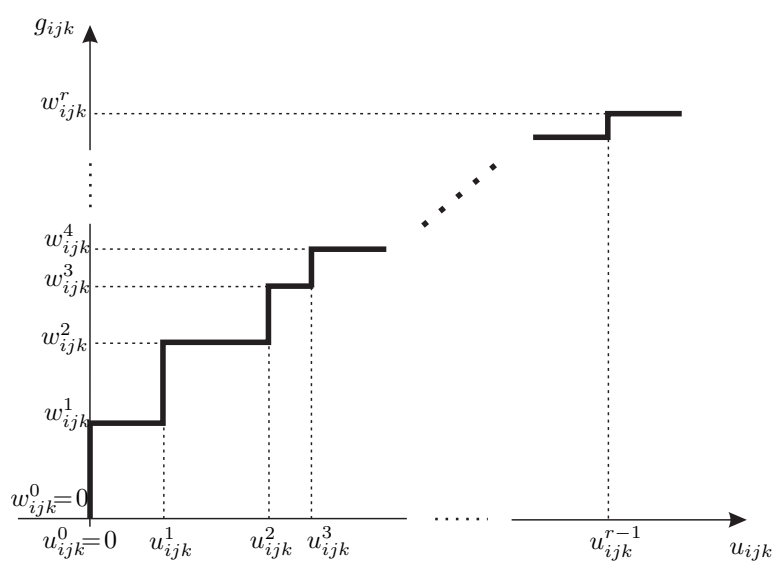

Fig. 1. Pictorial representation of a transportation cost function.

In this paper, instead of (8), the transportation costs are taken into account via a function $h_{i j k}:\{0,1\}^{r} \rightarrow \mathbb{R}$ and binary variables $y_{i j k}^{l}(t) \in\{0,1\}, i \in I(j), j=1,2, \ldots, N-$ $1, k \in K(i) \cap K(j), l=1,2, \ldots, r$, that are used to represent the various steps described for the function $g_{i j k}(\cdot)$. In other words, we consider the following transportation cost function:

$$
\begin{aligned}
& h_{i j k}\left[y_{i j k}^{1, r}(t)\right]=\sum_{l=1}^{r}\left(w_{i j k}^{l}-w_{i j k}^{l-1}\right) y_{i j k}^{l}(t), \\
& i \in I(j), \quad j=1,2, \ldots, N-1, \quad k \in K(i) \cap K(j)
\end{aligned}
$$

where $t=0,1, \ldots, T-1$ and $y_{i j k}^{1, r}(t) \triangleq \operatorname{col}\left[y_{i j k}^{1}(t)\right.$, $\left.y_{i j k}^{2}(t), \ldots, y_{i j k}^{r}(t)\right] \in\{0,1\}^{r}$. Let $\underline{y}_{t} \triangleq \operatorname{col}\left[y_{i j k}^{l}(t), i \in\right.$ $I(j), j=1,2, \ldots, N-1, k \in K(i) \cap K(j), l=$ $1,2, \ldots, r] \in\{0,1\}^{r m^{\prime}}$. Such variables take their values according to the following equation:

$$
\begin{aligned}
& y_{i j k}^{l}(t)=\left\{\begin{array}{l}
1 \text { if } u_{i j k}>u_{i j k}^{l-1}(t) \\
0 \text { otherwise }
\end{array}\right. \\
& i \in I(j), \quad j=1,2, \ldots, N-1, \quad k \in K(i) \cap K(j), \\
& l=1,2, \ldots, r
\end{aligned}
$$


where $t=0,1, \ldots, T-1$. Equation (10) can be taken into account by means of the following constraints:

$$
u_{i j k}(t) \leq M y_{i j k}^{l}(t)+u_{i j k}^{l-1}
$$$$
u_{i j k}(t)>M\left[y_{i j k}^{l}(t)-1\right]+u_{i j k}^{l-1}
$$

$i \in I(j), j=1,2, \ldots, N-1, k \in K(i) \cap K(j), l=1,2, \ldots, r$

where $t=0,1, \ldots, T-1$ and $M$ is a very large positive constant. Constraint (11a) is equivalent to $u_{i j k}(t) \leq u_{i j k}^{l-1}$ if $y_{i j k}^{l}(t)=0$, whereas it is trivially satisfied if $y_{i j k}^{l}(t)=1$. Constraint (11b) is equivalent to $u_{i j k}(t)>u_{i j k}^{l-1}$ if $y_{i j k}^{l}(t)=$ 1 , whereas it is trivially satisfied if $y_{i j k}^{l}(t)=0$.

To sum up, we consider the following cost function as tradeoff between inventory and transportation costs:

$$
\begin{aligned}
& J\left(\underline{x}_{0}^{T}, \underline{u}_{0}^{T-1}, \underline{y}_{0}^{T-1}\right)=c_{1} \sum_{t=1}^{T} \alpha^{t} \sum_{i=1}^{N-1} \sum_{k \in K(i)} z_{i k}(t) \\
& +c_{2} \sum_{t=0}^{T-1} \beta^{t} \sum_{j=1}^{N-1} \sum_{i \in I(j)} \sum_{k \in K(i) \cap K(j)} h_{i j k}\left[y_{i j k}^{1, r}(t)\right]
\end{aligned}
$$

where $\alpha \in(0,1]$ and $\beta \in(0,1]$ are discount coefficients; $c_{1}>0$ and $c_{2}>0$ are weighting constants.

Given $\underline{x}_{0}$ and $\underline{\hat{d}}_{t}$ for $t=0,1, \ldots, T-1$, we shall address the problem of minimizing the cost function (12) when the uncertainties on the external demands lead the system to the least favorable case, i.e., we state the following problem.

Problem 1. Find $\underline{x}_{1}^{T} \in \mathbb{R}_{+}^{n T}, \underline{u}_{0}^{T-1} \in \mathbb{R}_{+}^{m T}, \underline{s} \in \mathbb{R}_{+}^{q}, \underline{\Omega} \in$ $\{1,2, \ldots, T-1\}^{m^{\prime}}$, and $\underline{y}_{0}^{T-1} \in\{0,1\}^{r m^{\prime} T}$ such that

$$
\max _{\underline{\tilde{d}}_{\min , 0}^{T-1} \leq \underline{\tilde{d}}_{0}^{T-1} \leq \underline{\tilde{d}}_{\max , 0}^{T-1}} J\left(\underline{x}_{0}^{T}, \underline{u}_{0}^{T-1}, \underline{y}_{0}^{T-1}\right)
$$

is minimized under the constraints given by (1), the r.h.s. of (2), (4), (5a), (5b), (6), (7), (11a), and (11b).

Problem 1 is of min-max mixed-integer type and involves constraints with both real and integer unknowns. Concerning integer unknowns, they can be divided into two subsets, corresponding to delivery cycle times $\underline{\Omega}$ and binary vector $y_{0}^{T-1}$ related to transportation costs. Constraints (5a) and (5b) structurally depend on the integer variables that are the components of $\underline{\Omega}$, which define both number and structure of such constraints. The solution of Problem 1 can be obtained by using a top-down tree-based search that allows one to split the original problem into subproblems organized in a tree until a leaf node is reached. Each branching is made on the specific choice of $\Omega$. The leaves of the tree are made up of min-max mixed-integer problems with linear constraints and minimization variables that include the components of the binary vector $\underline{y}_{0}^{T-1}$, whereas $\underline{\Omega}$ is fixed. No problems to be solved are associated with the intermediate nodes, which are used only to select the values of the components of $\underline{\Omega}$. Each of such problems can be cast as follows.

Problem 1'. Given $\underline{\Omega} \in\{1,2, \ldots, T-1\}^{m^{\prime}}$, find $\underline{x}_{1}^{T} \in$ $\mathbb{R}_{+}^{n T}, \underline{u}_{0}^{T-1} \in \mathbb{R}_{+}^{m T}, \underline{s} \in \mathbb{R}_{+}^{q}$, and $\underline{y}_{0}^{T-1} \in\{0,1\}^{r m^{\prime} T}$ such that

$$
\max _{\underline{\tilde{d}}_{\min , 0}^{T-1} \leq \underline{\tilde{d}}_{0}^{T-1} \leq \underline{\tilde{d}}_{\max , 0}^{T-1}} J\left(\underline{x}_{0}^{T}, \underline{u}_{0}^{T-1}, \underline{y}_{0}^{T-1}\right)
$$

is minimized under the constraints given by (1), the r.h.s. of (2), (4), (5a), (5b), (6), (7), (11a), and (11b).

The tree that is obtained by solving the various Problems $1^{\prime}$ is composed of nodes generated on the basis of the selection of each component of $\Omega$ among the possible values from 1 to $T-1$ (see Fig. 2). The number of the leaves of such a tree is equal to $(T-1)^{m^{\prime}}$. In order to reduce the computational burden, a pruning strategy can be applied that consists in eliminating the leaves corresponding to larger values of a delivery cycle time for which the infeasibility of the related min-max problem has been already tested.

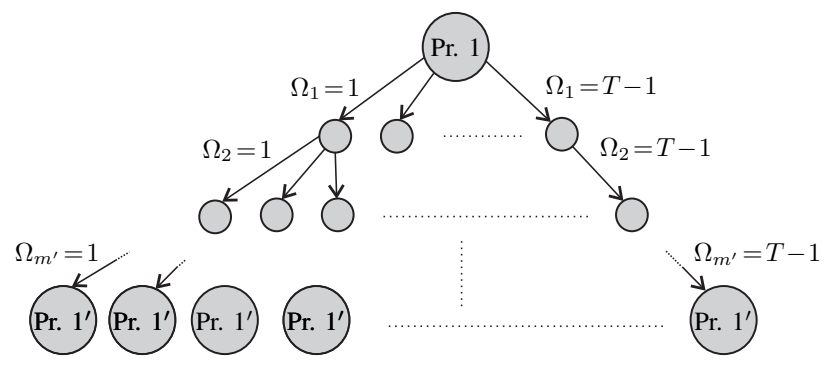

Fig. 2. Tree structure for the solution of Problem 1.

\section{TACTICAL CONTROL OF DCs Via MPC}

Since the actual customers' demands may be quite different from their corresponding forecasts available in the strategic planning, the resulting strategic policy can turn out to be inadequate to satisfy the demands during on-line operations. Thus, an adjustment of the control inputs during operations is well-suited to increasing the performances of the DC according to a tactical management strategy. Among the various alternatives to address the design of such a strategy, here we focus on the MPC paradigm, which consists in finding at each time step the control inputs that solve a receding-horizon optimal control problem of length $T_{p}$ (with $T_{p}<T$ ) and in applying only the first control action. The prediction and optimization steps are repeated at the successive time instants with an one-step forward shift of the sliding window and by using new (and possibly more precise) forecasts of the demands. In this case, the external demands have to be predicted only from time $t$ to time $t+T_{p}-1$, and not for all $t=0,1, \ldots, T-1$ as in the strategic case. Thus, now the predictions are assumed to be without uncertainty.

At each time $t=0,1, \ldots, T-1$, an open-loop finitehorizon optimal control problem is solved and the first optimal control input is applied instantaneously, thus turning into a closed-loop control action. Safety stocks and delivery cycle times are chosen to be equal to the corresponding optimal values obtained by solving Problem 1 in the strategic setting, denoted by $\underline{\bar{s}}^{\circ}$ and $\underline{\Omega}^{\circ}$, respectively. The goal of the tactical approach is to find the control inputs that minimize, at each time $t=0,1, \ldots, T-1$, the following cost function:

$J_{t}\left(\underline{x}_{t}^{t+T_{p}}, \underline{u}_{t}^{t+T_{p}-1}, \underline{y}_{t}^{t+T_{p}-1}\right)=\sum_{\tau=t+1}^{t+T_{p}}\left\{c_{1} \alpha^{\tau-t-1} \sum_{i=1}^{N-1} \sum_{k \in K(i)} z_{i k}(\tau)\right.$ 


$$
\begin{aligned}
& \left.+c_{3} \gamma^{\tau-t-1} \sum_{i=1}^{N-1} \sum_{k \in K(i)} \theta_{i k} P_{i k}\left[z_{i k}(\tau)\right]+c_{4} \delta^{\tau-t-1} \sum_{i \in \mathcal{S}} \sum_{k \in K(i)} b_{i k}(\tau)\right\} \\
& +\sum_{\tau=t}^{t+T_{p}-1} c_{2} \beta^{\tau-t} \sum_{j=1}^{N-1} \sum_{i \in I(j)} \sum_{k \in K(i) \cap K(j)} h_{i j k}\left[y_{i j k}^{1, r}(\tau)\right]
\end{aligned}
$$

where $\alpha \in(0,1], \beta \in(0,1], \gamma \in(0,1]$, and $\delta \in(0,1]$ are discount coefficients; $c_{1}, c_{2}, c_{3}$, and $c_{4}$ are positive weighting constants; $h_{i j k}:\{0,1\}^{r} \rightarrow \mathbb{R}$ is the function that accounts for the transportation costs as in (9); $P_{i k}: \mathbb{R} \rightarrow \mathbb{R}$ is a penalization function for the z-variable $z_{i k}(t)$, whose aim is to penalize the consumption of the safety stocks fixed in the strategic planning, and $\theta_{i k}>0$ is the corresponding weight. A possible choice for the function $P_{i k}(\cdot)$ is the following:

$$
P_{i k}\left(z_{i k}\right)= \begin{cases}\left(-z_{i k}+\bar{s}_{i k}^{\circ}\right)^{v} & \text { if } 0 \leq z_{i k} \leq \bar{s}_{i k}^{\circ} \\ 0 & \text { if } \quad z_{i k}>\bar{s}_{i k}^{\circ} .\end{cases}
$$

where $v>0$ is chosen basing on the required degree of smoothness for $z_{i k}=\bar{s}_{i k}^{\circ}$.

Once fixed the safety stocks $\underline{\bar{s}}^{\circ}$ and delivery cycle times $\bar{\Omega}^{\circ}$, given the state vector $\underline{x}_{t}$ and demand predictions $\underline{\hat{d}}_{t}^{t+T_{p}-1}$ at each time $t=0,1, \ldots, T-1$, we deal with the following problem.

Problem 2. Find $\underline{x}_{t+1}^{t+T_{p}} \in \mathbb{R}_{+}^{n T_{p}}, \underline{u}_{t}^{t+T_{p}-1} \in \mathbb{R}_{+}^{m T_{p}}$, and $\underline{y}_{t}^{t+T_{p}-1} \in\{0,1\}^{r m^{\prime} T_{p}}$ such that

$$
J_{t}\left(\underline{x}_{t}^{t+T_{p}}, \underline{u}_{t}^{t+T_{p}-1}, \underline{y}_{t}^{t+T_{p}-1}\right)
$$

is minimized under the constraints given by (1), the r.h.s. of (2), (5a), (5b), (11a), and (11b).

In this case, $\underline{\hat{d}}_{t}$ is assumed to be a perfect prediction of $\underline{d}_{t}$, and thus in general different from that available in the strategic setting. Problem 2 is of mixed-integer programming type because of the presence of the binary variables $\underline{y}_{t}^{t+T_{p}-1}$. As previously noted, such binary variables are not required in [1] since, instead of the function $h_{i j k}(\cdot)$, the step-increasing function $g_{i j k}(\cdot)$ reported in (8) is used to account for the transportation costs. Clearly, this makes the cost (13) nondifferentiable, thus turning into the necessity of employing only nonderivative optimization methods. By contrast, the approach proposed here does not suffer from such a limitation, but at the price of dealing with binary variables.

Problem 2 differs from Problem 1 for various reasons. First of all, it has to be solved on line with fixed safety stocks and delivery cycle times. Since the delivery cycle times are given, Problem 2 does not require the previously described tree search. Thus, the computational effort required on line is reduced as compared with that of Problem 1. Another remarkable difference between the strategic and tactical levels concerns the safety stock violation constraints (4) of Problem 1, which are removed in Problem 2 and replaced by terms that penalize the use of safety stocks in the cost function. During on-line operations, such quantities of goods are available upon request to deal with unpredictable demands, thus possibly preventing from stockouts. Finally, in the MPC tactical setting we assume to have at disposal very precise demand predictions and deal with optimization problems of much smaller dimensions than those of Problems $1^{\prime}$.

\section{Simulation Results}

As an example of application, let us consider the singleitem DC made up of 4 nodes depicted in Fig. 3. Since such a DC is only with a single item, in the following we shall drop the subscript $k$ from all the variables and functions. We evaluated the DC performance over one year, where each bucket corresponds to one week (i.e., we chose $T=52$ ). All the simulations were performed in Matlab using also the Tomlab optimization software [10].

The considered DC turns out to be governed by a dynamic equation with $\underline{x}_{t} \in \mathbb{R}^{15}, t=0,1, \ldots, T, \underline{u}_{t} \in \mathbb{R}^{7}, t=$ $0,1, \ldots, T-1, \underline{y}_{t} \in \mathbb{R}^{15}, t=0,1, \ldots, T-1$, and $\underline{d}_{t} \in \mathbb{R}^{2}$, $t=0,1, \ldots, T-1$.

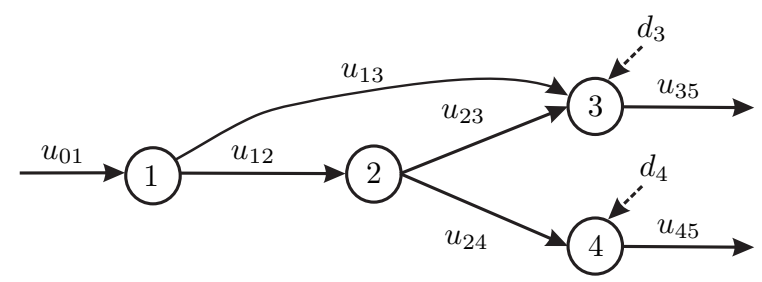

Fig. 3. Graph of the single-item DC considered in the simulations.

Concerning the strategic level, in order to reduce the computational complexity of the tree generation, the maximum value of the delivery cycle times was chosen to be equal to 4. Basing on such a tree structure, the solution of the related Problem 1 was found by searching for the optimal amounts of goods to transfer from node to node, safety stocks, and delivery cycle times using the information on long-term forecasts of the external demands given only by the uncertainty bands of the predictions of the demands $d_{3}(t)$, and $d_{4}(t)$ for $t=0,1, \ldots, T-1$ (see the dashed lines in Fig. 4, whereas the continuous lines represent the actual demands used at the tactical level). As regards the cost function, we chose all the coefficients $\alpha, \beta, c_{1}$, and $c_{2}$ equal to 1 and the same function $h:\{0,1\}^{r} \rightarrow \mathbb{R}$ for all $i \in I(j), j=1,2, \ldots, 4$, with $r=3, w_{i j}^{1}=10, w_{i j}^{2}=20$, $w_{i j}^{3}=30, u_{i j}^{1}=100$, and $u_{i j}^{2}=200$. Other details are briefly summarized in Table I. The solution of Problem 1 provided the optimal delivery cycle times and safety stocks $\underline{\bar{\Omega}}^{\circ}=\operatorname{col}(4,2,2,1,1)$ and $\underline{\bar{s}}^{\circ}=\operatorname{col}(1861,742,10,113)$, respectively.

As for the tactical level, we solved Problem 2 to compute the optimal control inputs $\underline{u}_{t}$ at each time bucket $t=$ $0,1, \ldots, T-1$ with MPC during on-line operations basing on the values $\underline{\bar{s}}^{\circ}$ and $\underline{\bar{\Omega}}^{\circ}$ obtained in the strategic setting. The transportation delays, as well as the initial and maximum stock levels of all the buffers were chosen to be equal to those reported in Table I. We chose $\underline{u}_{\min , t}=\underline{0}$ for all $t=0,1, \ldots, T-1$ and the components of $\underline{u}_{\max , t}$ equal to the corresponding forecast flows determined in the strategic 

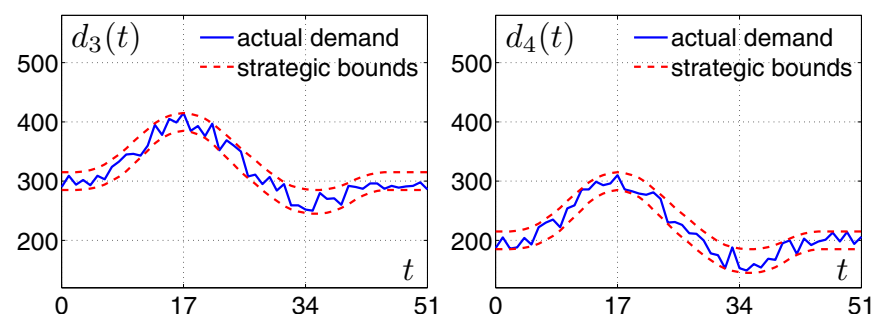

Fig. 4. External demands used at the tactical level and demand prediction bands of the strategic planning.

TABLE I

PARAMETERS AND VARIABLES OF THE SIMULATIONS.

\begin{tabular}{ccccc}
\hline Node $i$ & Node $j$ & $\tau_{i j}$ & $u_{i j}^{\min }(t)$ & $u_{i j}^{\max }(t)$ \\
\hline 0 & 1 & 2 & 1700 & 3200 \\
1 & 2 & 2 & 600 & 1200 \\
1 & 3 & 3 & 200 & 800 \\
2 & 3 & 1 & 100 & 300 \\
2 & 4 & 1 & 100 & 300 \\
& & & & \\
\hline Node $i$ & $z_{i}(0)$ & $z_{i}^{\max }$ & $z_{i}^{\text {down }}$ & $z_{i}^{\text {up }}$ \\
\hline 1 & 10000 & 10000 & 2000 & 5000 \\
2 & 5000 & 10000 & 1000 & 3000 \\
3 & 200 & 3000 & 10 & 500 \\
4 & 200 & 3000 & 10 & 500 \\
\hline
\end{tabular}

case plus $15 \%$. In the cost function, we chose $\theta_{i}=1$ for all $i=1,2, \ldots, 4$, all the coefficients $\alpha, \beta, c_{1}, c_{2}$, and $c_{3}$ equal to $1, c_{4}=100$, and $v=2$. Such a choice of the coefficients allows one to satisfy more promptly the demands by penalizing much more the backlogs rather than the other terms.

The evaluation of the effectiveness of the considered approach was performed by means of a key performance index (KPI) defined as follows:

$$
\mathrm{KPI} \triangleq c_{1} \sum_{t=0}^{T} \sum_{i=1}^{4} z_{i}(t)+c_{2} h\left[y_{i j}^{1, r}(t)\right]+c_{4} \sum_{t=0}^{T} \sum_{i=3}^{4} b_{i}(t)
$$

where the weights $c_{1}, c_{2}$, and $c_{4}$ are the same coefficients used in the cost function (13). Such an index is made up of the weighted sum of the costs related to the inventory levels, the transportation costs, and the costs of unsatisfied demands.

We compared the results obtained by solving Problem 2 with the approach presented in [1], where the step-increasing expression (8) was used for the transportation cost function without introducing the binary variables $\underline{y}_{t}, t=0,1, \ldots, T-$ 1 . Different lengths of the sliding window were considered, i.e., we chose $T_{p}$ equal to 1,2 , and 3 . The use of larger values of $T_{p}$ may be critical since it would entail on-line predictions of the external demands over a too long horizon, for which the assumption of having at disposal exact forecasts may be difficult to hold. The KPIs obtained in the various cases are reported in Table II.

The simulation results show that, as the control horizon $T_{p}$ increases, the KPI decreases since more effective control ac-
TABLE II

KPIS OBTAINED WITH DIFFERENT PREDICTIVE CONTROL HORIZONS.

\begin{tabular}{ccc}
\hline$T_{p}$ & mixed-integer & relaxed \\
\hline 1 & $4.78 \cdot 10^{5}$ & $4.80 \cdot 10^{5}$ \\
2 & $4.76 \cdot 10^{5}$ & $4.79 \cdot 10^{5}$ \\
3 & $2.83 \cdot 10^{5}$ & $2.99 \cdot 10^{5}$ \\
\hline
\end{tabular}

tions are computed because of higher amount of information about the future is taken into account. As compared with the approach of [1], here the obtained results in terms of values of the KPI are better; however, an increased computational effort is needed to perform the optimization since we deal with mixed-integer programming problems rather than with real programming ones.

The main drawback of the approach presented in this paper is the fact that the number of binary variables increases with the number of links, the number of levels of the transportation cost function $h_{i j k}(\cdot)$, and the length of the control horizon $T_{p}$ in Problem 2. Thus, even if the approach presented here allows one to obtain better results with respect to the relaxation-based approach adopted in [1], it may turn out to be unfeasible, especially for DCs with many nodes and links, as well as a high number of levels in the transportation cost function and a large length of the control horizon $T_{p}$. In this simple example, the instances of Problem 2 corresponding to $T_{p}=1, T_{p}=2$, and $T_{p}=3$ were characterized by 15,30 , and 45 binary variables, respectively. In order to tackle the computational difficulties, heuristic techniques will be the subject of future works with the goal of dealing with problems of higher dimensionality.

\section{REFERENCES}

[1] A. Alessandri, M. Gaggero, and F. Tonelli, "Min-max and predictive control for the management of distribution in supply chains," IEEE Trans. Contr. Syst. Technol., 2011, to appear, DOI:10.1109/TCST.2010.2076283.

[2] O. Özer, "Replenishment strategies for distribution systems under advance demand information," Manag. Sci., vol. 49, no. 3, pp. 255$272,2003$.

[3] H. Wee and P. Yang, "The optimal and heuristic solutions of a distribution network," Eur. J. Oper. Res., vol. 158, no. 3, pp. 626632, 2004.

[4] T. Wang and B. Toktay, "Inventory management with advance demand information and flexible delivery," Manag. Sci., vol. 54, no. 4, pp. 716$732,2008$.

[5] A. Alessandri, C. Cervellera, M. Cuneo, M. Gaggero, and G. Soncin, "Management of logistics operations in intermodal terminals by using dynamic modelling and nonlinear programming," Maritime Economics \& Logistics, vol. 11, no. 1, pp. 58-76, 2009.

[6] A. Alessandri, C. Cervellera, M. Cuneo, and M. Gaggero, "Nonlinear model predictive control for resource allocation in the management of intermodal container terminals," in Nonlinear Model Predictive Control - Towards New Challenging Applications, L. Magni et al., Ed. Springer-Verlag Berlin Heidelberg.

[7] L. Wolsey, Integer Programming. John Wiley, New York, 1998.

[8] D. Axehill, L. Vandenberghe, and A. Hansson, "Convex relaxations for mixed integer predictive control," Automatica, vol. 46, no. 9, pp. $1540-1545,2010$.

[9] Q. Zhai, X. Guan, and F. Gao, "Optimization based production planning with hybrid dynamics and constraints," IEEE Trans. Automat. Cont., vol. 55, no. 12, pp. $2778-2792,2010$.

[10] K. Holmström, "The TOMLAB optimization environment in Matlab," Advanced Modeling and Optimization, vol. 1, no. 1, pp. 47-69, 1999. 